\begin{tabular}{|c|l|}
\hline Title & The determinant of a hy pergeometric period matrix \\
\hline Author(s) & Douai, A.; Terao, H. \\
\hline Citation & Hokkaido University Preprint Series in Mathematics, 345, 1-20 \\
\hline Issue Date & 1996-9-1 \\
\hline DOI & 10.14943/83491 \\
\hline Doc URL & http://hdl.handle.net/2115/69095 \\
\hline Type & bulletin (article) \\
\hline File Information & pre345.pdf \\
\hline
\end{tabular}

Instructions for use 
The determinant of a hypergeometric period matrix
A. Douai and H. Terao

Series \#345. September 1996 


\section{HOKKAIDO UNIVERSITY PREPRINT SERIES IN MATHEMATICS}

\#321 M. Ohnuma, Axisymmetric solutions and singular parabolic equations in the theory of viscosity solutions, 26 pages. 1995.

\#322 T. Nakazi, An outer function and several important functions in two variables, 12 pages. 1995.

\#323 N. Kawazumi, An infinitesimal approach to the stable cohomology of the moduli of Riemann surfaces, 22 pages. 1995.

\#324 A. Arai, Factorization of self-adjoint operators by abstract Dirac operators and its application to second quantizations on Boson Fermion Fock spaces, 15 pages. 1995.

\#325 K. Sugano, On strongly separable Frobenius extensions, 11 pages. 1995.

\#326 D. Lehmann and T. Suwa, Residues of holomorphic vector fields on singular varieties, 21 pages. 1995.

\#327 K. Tsutaya, Local regularity of non-resonant nonlinear wave equations, 23 pages. 1996.

\#328 T. Ozawa and Y. Tsutsumi, Space-time estimates for null gauge forms and nonlinear Schrödinger equations, 25 pages. 1996.

\#329 O. Ogurisu, Anticommutativity and spin 1/2 Schrödinger operators with magnetic fields, 12 pages. 1996.

\#330 Y. Kurokawa, Singularities for projections of contour lines of surfaces onto planes, 24 pages. 1996.

\#331 M.-H. Giga and Y. Giga, Evolving graphs by singular weighted curvature, 94 pages. 1996.

\#332 M. Ohnuma and K. Sato, Singular degenerate parabolic equations with applications to the $p$-laplace diffusion equation, 20 pages. 1996.

\#333 T. Nakazi, The spectra of Toeplitz operators with unimodular symbols, 9 pages. 1996.

\#334 B. Khanedani and T. Suwa, First variation of horomorphic forms and some applications, 11 pages. 1996.

\#335 J. Seade and T. Suwa, Residues and topological invariants of singular holomorphic foliations ${ }^{1}, 28$ pages. 1996.

\#336 Y. Giga, M.E. Gurtin and J. Matias, On the dynamics of crystalline motions, 67 pages. 1996.

\#337 I. Tsuda, A new type of self-organization associated with chaotic dynamics in neural networks, 22 páges. 1996.

\#338 F. Hiroshima, A scaling limit of a Hamiltonian of many nonrelativistic particles interacting with a quantized radiation field, 34 pages. 1996 .

\#339 N. Tominaga, Analysis of a family of strongly commuting self-adjoint operators with applications to perturbed Dirac operators, 29 pages. 1996.

\#340 A. Inoue, Abel-Tauber theorems for Fourier-Stieltjes coefficients, 17 pages. 1996.

\#341 G. Ishikawa, Topological classification of the tangent developables of space curves, 19 pages. 1996.

\#342 Y. Shimizu, A remark on estimates of bilinear forms of gradients in Hardy space, 8 pages. 1996.

\#343 N. kawazumi and S. Morita, The primary approximation to the cohomology of the moduli space of curves and cocycles for the stable characteristic classes, 11 pages. 1996.

\#344 M.-H. Giga and Y. Giga, A subdifferential interpretation of crystalline motion under nonuniform driving force, 18 pages. 1996 . 


\title{
The determinant of a hypergeometric period matrix
}

\author{
ANTOINe DouaI \\ Unité associée au CNRS 168, Université de Nice, Parc Valrose, F-06108 Nice Cedex 2 FRANCE \\ HiRoAKI TERAO * \\ Department of Mathematics, University of Wisconsin, Madison, WI 53706 USA
}

\section{Introduction}

Let $f_{1}, \ldots, f_{p}$ be polynomials with real coefficients of degree one which define an arrangement $\mathcal{A}$ of hyperplanes in $\mathbb{R}^{n}$ or $\mathbb{C}^{n}$. Let $\alpha_{1}, \ldots, \alpha_{p}$ complex numbers. Let $U_{\alpha}=f_{1}^{\alpha_{1}} \ldots f_{p}^{\alpha_{p}}$. Varchenko ([V1, Theorem 1.1], [V2]) calculated, for arrangements of hyperplanes in general position, the determinant of a (period) matrix $\operatorname{PM}(\mathcal{A}, \alpha)$ whose entries are (hypergeometric) integrals $\int_{\Delta} U_{\alpha} \phi$, where $\Delta$ runs over the set $\operatorname{Ch}(\mathcal{A})$ of bounded connected components of $\mathbb{R}^{n}-\bigcup_{i=1}^{p}\left\{f_{i}=0\right\}$ and the $n$-form $\phi$ runs over the set $\Phi^{p}(\mathcal{A})=\left\{\alpha_{i_{1}} \cdots \alpha_{i_{n}} d f_{i_{1}} / f_{i_{1}} \wedge \cdots \wedge\right.$ $\left.d f_{i_{n}} / f_{i_{n}} \mid 1<i_{1}<\cdots<i_{n} \leq p\right\}$. Since $|C h(\mathcal{A})|=\left|\Phi^{p}(\mathcal{A})\right|=\left(\begin{array}{c}p-1 \\ n\end{array}\right)$, the period matrix $\operatorname{PM}(\mathcal{A}, \alpha)$ is of size $\left(\begin{array}{c}p-1 \\ n\end{array}\right) \times\left(\begin{array}{c}p-1 \\ n\end{array}\right)$. The formula by Varchenko expresses the determinant of $\operatorname{PM}(\mathcal{A}, \alpha)$ by the product of critical values and a certain function, called the Beta function $B(\mathcal{A}, \alpha)$ of the arrangement. The Beta function $B(\mathcal{A}, \alpha)$ is explicitly given as an alternating product of Gamma functions whose arguments are appropriate linear combinations of the parameters $\alpha_{i}$. In the general case, Varchenko conjectured an analogous explicit formula [V2, 6.3 Fundamental conjecture] and proved it for normal-crossing arrangements [V1, Theorem 1.4] and arrangements in general position at infinity [V2, Theorem 6.1]. Note that this determinant can also regarded as "Wronskian" of a certain system of partial differential equations (cf. for example [Ki]).

More recently, F. Loeser and C. Sabbah ([LS]), gave a general formula for such a determinant. In this formula enters the characteristic polynomial of some monodromies associated with the family $f_{1}, \cdots, f_{p}$ of polynomials. Since the bases of cycles and forms are not specified, there are some unknowns in their result and our purpose is to remove them. In order to do that, we use the $\beta$ nbc-bases of the cohomology defined in [FT, Theorem 3.6]. The calculation of the determinant of the period matrix in a $\beta \mathrm{nbc}$-basis can be done by using recurrence (deletion-restriction) and by studying zeros and poles of the determinant in terms

*second author partially supported by NSF Grant DMS950445i 
of the non-resonance set. In the end, we show that the conjecture announced by Varchenko is true for any arrangement for an arbitrary $\beta$ nbc-basis.

\section{Arrangements}

In this section we review results from $[\mathrm{FT}]$.

\section{1}

Let $f_{1}, \ldots, f_{p}$ be linear polynomials defined on the $n$-dimensional complex affine space $V$. Let $I$ denote $\{1, \ldots, p\}$ and $\mathcal{A}$ be the arrangement $\left\{H_{i}\right\}_{i \in I}$ where $H_{i}=$ ker $f_{i}$ is the hyperplane defined by $f_{i}$.

Definition 2.1.1 An edge of $\mathcal{A}$ is a nonempty intersection of some of its hyperplanes.

Let $L(\mathcal{A})$ denote the set of all these edges. $L(\mathcal{A})$ is partially ordered by reverse inclusion. The maximal elements of $L(\mathcal{A})$ all have the same codimension, which we denote by $r$. Agree that $V \in L(\mathcal{A})$ which is the unique minimum element.

Definition 2.1.2 (i) $\mathcal{A}$ is said to be essential if $r=n$ (in particular $|I| \geq n$ ). (ii) $\mathcal{A}$ is said to be real if the polynomials $f_{i}$ all have real coefficients.

Definition 2.1.3 An arrangement $\mathcal{A}$ is said to be in general position if, for all subarrangement $\left\{H_{i_{1}}, \cdots, H_{i_{k}}\right\}$ of $\mathcal{A} \operatorname{codim}\left(H_{i_{1}} \cap \cdots \cap H_{i_{k}}\right)=k$ if $1 \leq k \leq n$ and $H_{i_{1}} \cap \cdots \cap H_{i_{k}}=\emptyset$ if $k>n$. An arrangement $\mathcal{A}$ is said to be in general position at infinity if, for all subarrangement $\left\{H_{i_{1}}, \cdots, H_{i_{k}}\right\}$ of $\mathcal{A}, H_{i_{1}} \cap \cdots \cap H_{i_{k}} \neq \emptyset$ for $k \leq n$. It is said to be normal if $\cup H$ is a normal crossing divisor in $V$.

Notation 2.1.4 (i) Let

$$
M(\mathcal{A})=V-\cup_{i \in I} H_{i}
$$

and, if $\mathcal{A}$ is real,

$$
M_{\mathbb{R}}(\mathcal{A})=M(\mathcal{A}) \cap V_{\mathbb{R}}
$$

where $V_{\mathbb{R}}$ denote the real part of $V$.

(ii) If $\mathcal{A}$ is real, let $\mathrm{Ch}(\mathcal{A})$ denote the set of all $n$-dimensional bounded components of $M_{\mathbb{R}}(\mathcal{A})$ and $\beta(\mathcal{A})$ its cardinality.

Until the end of this paper we suppose $\mathcal{A}$ real and essential. 


\subsection{Linear orders}

Let $i_{0} \in I$. We define a linear order $<_{i_{0}}$ in $\mathcal{A}$ putting $H_{i}<_{i_{0}} H_{j}$ if $i<j, i, j \neq i_{0}$, and $H_{i}<_{i_{0}} H_{i_{0}}$ for all $i \in I-\left\{i_{0}\right\}$.

Remark 2.2.1 $(i)<_{p}$ is the standard order defined in [OT, page 67].

(ii) If $\mathcal{B} \subset \mathcal{A}$ is a subarrangement which do not contains $H_{i_{0}}$, it inherits the standard order.

\subsection{Non-resonant weights}

Let $F \in L(\mathcal{A})-\{V\}$ be an edge. Define

$$
I(F)=\left\{i \in I \mid F \subseteq H_{i}\right\} .
$$

Definition 2.3.1 The edge $F$ is called dense if the arrangement

$$
\left\{H_{i} \mid i \in I(F)\right\}
$$

is not decomposable [STV, Section 2], that is, the arrangement is not a product of two nonempty arrangements.

Let $\mathbb{P}^{n}$ be complex projective space, which is a compactification of $V=\mathbb{C}^{n}$. Define the arrangement $\mathcal{A}_{\infty}$ of projective hyperplanes by

$$
\mathcal{A}_{\infty}=\left\{\bar{H}_{1}, \bar{H}_{2}, \ldots, \bar{H}_{p}, \bar{H}_{\infty}\right\}
$$

where $\bar{H}_{i}$ is the projective closure of $H_{i}(1 \leq i \leq p)$ and $\bar{H}_{\infty}=\mathbb{P}^{n}-\mathbb{C}^{n}$. Let $L\left(\mathcal{A}_{\infty}\right)$ be the collection of nonempty intersections of projective hyperplanes in $\mathcal{A}_{\infty}$. Define $L_{-}\left(\mathcal{A}_{\infty}\right)$ (resp. $L_{+}\left(\mathcal{A}_{\infty}\right)$ ) to be the set of edges of $L\left(\mathcal{A}_{\infty}\right)$ contained (resp. not contained) in $\bar{H}_{\infty}$. Then $L\left(\mathcal{A}_{\infty}\right)=L_{-}\left(\mathcal{A}_{\infty}\right) \cup L_{+}\left(\mathcal{A}_{\infty}\right)$ (disjoint). Cover $\mathbb{P}^{n}$ by the standard affine opens $U_{0}, U_{1}, \ldots, U_{n}$, each of which is isomorphic to $\mathbb{C}^{n}$. Let $\mathcal{A}_{i}(0 \leq i \leq n)$ be the arrangement in $U_{i} \simeq \mathbb{C}^{n}$ obtained by restricting each projective hyperplane in $\mathcal{A}_{\infty}$ to $U_{i}$. Let $F \in$ $L\left(\mathcal{A}_{\infty}\right)-\left\{\mathbb{P}^{n}\right\}$. We say that $F$ is dense if $F \cap U_{i}$ is dense in $\mathcal{A}_{i}$ for $0 \leq i \leq n$ with $F \cap U_{i} \neq \emptyset$. (cf. [STV, Section 3].)

Let $\bar{I}=\{1, \ldots, p, \infty\}$. For $F \in L\left(\mathcal{A}_{\infty}\right)$ define

$$
\bar{I}(F)=\left\{i \in \bar{I} \mid F \subseteq \bar{H}_{i}\right\} .
$$

To each polynomial $f_{i}$ (and therefore to each hyperplane $H_{i}$ ), one associates a complex number $\alpha_{i}$. These numbers are called weights. Define $\alpha_{\infty}=-\sum_{i=1}^{p} \alpha_{i}$. For $F \in L\left(\mathcal{A}_{\infty}\right)-$ $\left\{\mathbb{P}^{n}\right\}$, let $\alpha(F)$ be the sum of $\alpha_{i}$ with $i \in \bar{I}(F)$. In other words, for $F \in L_{+}\left(\mathcal{A}_{\infty}\right)$,

$$
\alpha(F)=\sum_{i \in I(F)} \alpha_{i}
$$


and, for $F \in L_{-}\left(\mathcal{A}_{\infty}\right)$,

$$
\alpha(F)=\alpha_{\infty}+\sum_{j \in \operatorname{In} \bar{I}(F)} \alpha_{i}=-\sum_{i \in I} \alpha_{i}+\sum_{j \in \operatorname{In} \bar{I}(F)} \alpha_{i}=-\sum_{j \in I-\bar{I}(F)} \alpha_{i} .
$$

Define the resonance set of $\mathcal{A}$ by

$$
\operatorname{Rsn}(\mathcal{A})=\left\{\alpha=\left(\alpha_{1}, \ldots, \alpha_{p}\right) \in \mathbb{C}^{p} \mid \alpha(F) \in \mathbb{Z} \text { for some dense edge } F \in L\left(\mathcal{A}_{\infty}\right)\right\}
$$

It is the union of a locally finite infinite family of hyperplanes.

Definition 2.3.2 We say that the weights $\alpha=\left(\alpha_{1}, \cdots, \alpha_{p}\right)$ are non-resonant if $\alpha \notin$ $\operatorname{Rsn}(\mathcal{A})$. In other words, the weights $\alpha$ are non-resonant if $\alpha(F) \notin \mathbb{Z}$ for each dense edge $F \in L\left(\mathcal{A}_{\infty}\right)$.

\section{$2.4 \beta$ nbc-bases}

Let $i_{0} \in I$. We define the linear order $<_{i_{0}}$ in $\mathcal{A}$.

A subset $\left\{H_{i}\right\}_{i \in J}$ of $\mathcal{A}$ is dependent if $\cap_{i \in J} H_{i} \neq \emptyset$ and $\operatorname{codim}\left(\cap_{i \in J} H_{i}\right)<|J|$. A subset of $\mathcal{A}$ which has nonempty intersection and is not dependent is called independant. Maximal independent sets are called bases. Every base has cardinality $n$.

A $k$-tuple $S=\left(H_{1}, \cdots, H_{k}\right)$ is a circuit if $\left(H_{1}, \cdots, H_{k}\right)$ is dependent and if, for each $l$, $1 \leq l \leq k$, the $(k-1)$-tuple $\left(H_{1}, \cdots, \widehat{H}_{l}, \cdots, H_{k}\right)$ is independent. A $k$-tuple $S$ is a broken circuit if there exists $H<_{i_{0}} \min (S)$ such that $\{H\} \cup S$ is a circuit, where $\min (S)$ denotes the minimal element of $S$ for $<_{i_{0}}$.

The collection of subsets of $\mathcal{A}$ having nonempty intersection and containing no broken circuits is denoted by $\mathrm{BC}$. BC consists of independent sets. Maximal (with respect to inclusion) elements of $\mathbf{B C}$ are bases of $\mathcal{A}$ called nbc-bases.

A nbc-basis $B=\left(H_{i_{1}}, \cdots, H_{i_{n}}\right)$ is ordered if $H_{i_{1}}<i_{0} H_{i_{2}}<i_{0} \cdots<i_{0} H_{i_{n}}$.

We denote by $\operatorname{nbc}_{i_{0}}(\mathcal{A})$ the set of all ordered nbc-bases of $\mathcal{A}$. We introduce a linear order in $\mathrm{nbc}_{i_{0}}(\mathcal{A})$ using the lexicographic order on the hyperplanes read from right to left.

Definition 2.4.1 $A$ basis $B$ is called $\beta$ nbc-basis if $B$ is a nbc-basis and if, for every $H \in B$, there exists $H^{\prime}<_{i_{0}} H$ such that $(B-\{H\}) \cup\left\{H^{\prime}\right\}$ is a base.

Notation 2.4.2 Denote by $\beta \mathrm{nbc}_{i_{0}}(\mathcal{A})$ the set of all $\beta$ nbc-bases, ordered by $<_{i_{0}}$.

Remark 2.4.3 (i) $\beta \mathrm{nbc}_{i_{0}}(\mathcal{A})$ inherits the order defined on $\mathrm{nbc}_{i_{0}}(\mathcal{A})$.

(ii) In what follows, we omit the index $i_{0}$ when the linear order on $\mathcal{A}$ is the standard order.

Definition 2.4.4 If $B=\left(H_{i_{1}}, \cdots, H_{i_{n}}\right) \in \beta \mathrm{nbc}_{i_{0}}(\mathcal{A})$, let

$$
F_{j}=\bigcap_{k=j+1}^{n} H_{i_{k}}
$$


for $0 \leq j \leq n-1$ and $F_{n}=V$. Define

$$
\xi(B)=\left(F_{0} \subset F_{1} \subset \cdots \subset F_{n}\right),
$$

which is a flag of affine subspaces of $V$ with $\operatorname{dim} F_{j}=j(0 \leq j \leq n)$. This flag $\xi(B)$ is called the $\beta$ nbc-flag associated with $B$.

Notation 2.4.5 For $\left(i_{1}, \cdots, i_{k}\right) \subseteq I, \omega_{i_{1} \ldots i_{k}}=d f_{i_{1}} / f_{i_{1}} \wedge \cdots \wedge d f_{i_{k}} / f_{i_{k}}$. If $F \in L(\mathcal{A})-\{V\}$, define

$$
I(F)=\left\{i \in I \mid F \subseteq H_{i}\right\}
$$

and

$$
\omega_{\alpha}(F, \mathcal{A})=\sum_{i \in I(F)} \alpha_{i} \omega_{i}
$$

For $B=\left(H_{i_{1}}, \cdots, H_{i_{n}}\right) \in \beta \mathrm{nbc}_{i_{0}}(\mathcal{A})$, let $\xi(B)=\left(F_{0} \subset F_{1} \subset \cdots \subset F_{n}\right)$ be the associated flag. Define

$$
\Xi(B, \mathcal{A})=\omega_{\alpha}\left(F_{0}, \mathcal{A}\right) \wedge \cdots \wedge \omega_{\alpha}\left(F_{n-1}, \mathcal{A}\right)
$$

Definition 2.4.6 If $\beta \mathrm{nbc}_{i_{0}}(\mathcal{A})=\left\{B_{1}, \cdots, B_{\beta(\mathcal{A})}\right\}$ and $\phi_{i}^{i_{0}}=\phi_{i}^{i_{0}}(\mathcal{A})=\Xi\left(B_{i}, \mathcal{A}\right)$, define

$$
\Phi^{i_{0}}(\mathcal{A})=\left\{\phi_{1}^{i_{0}}, \cdots, \phi_{\beta(\mathcal{A})}^{i_{0}}\right\} .
$$

Define

$$
I_{i_{0}}^{\prime}=I-\left\{i_{0}\right\}
$$

and

$$
\mathcal{A}_{i_{0}}^{\prime}=\left\{H_{i}^{\prime}\right\}_{i \in I_{i_{0}}^{\prime}} \text { with } H_{i}^{\prime}=H_{i}
$$

Let

$$
\mathcal{A}_{i_{0}}^{\prime \prime}=\left\{H_{i} \cap H_{i_{0}} \mid i \in I_{i_{0}}^{\prime}, H_{i} \cap H_{i_{0}} \neq \emptyset\right\} \text {. }
$$

Then $\mathcal{A}_{i_{0}}^{\prime \prime}$ is an arrangement of hyperplanes in $H_{i_{0}}$. The linear order on $\mathcal{A}_{i_{0}}^{\prime}$ is inherited from $\mathcal{A}$. If $H^{\prime \prime} \in \mathcal{A}_{i_{0}}^{\prime \prime}$, let $\nu\left(H^{\prime \prime}\right)$ be the smallest hyperplane of $\mathcal{A}_{i_{0}}^{\prime}$ containing $H^{\prime \prime}$. We order $\mathcal{A}_{i_{0}}^{\prime \prime}$ setting $H^{\prime \prime}<K^{\prime \prime}$ if and only if $\nu\left(H^{\prime \prime}\right)<_{i_{0}} \nu\left(K^{\prime \prime}\right)$.

Remark 2.4.7 $\mathcal{A}_{i_{0}}^{\prime}$ and $\mathcal{A}_{i_{0}}^{\prime \prime}$ are equipped with standard orders.

Let

$$
I_{i_{0}}^{\prime \prime}=\left\{i \in I_{i_{0}}^{\prime} \mid H_{i}=\nu\left(H^{\prime \prime}\right) \text { for some } H^{\prime \prime} \in \mathcal{A}_{i_{0}}^{\prime \prime}\right\} \text {. }
$$

To each $i \in I_{i_{0}}^{\prime}$ (or $i \in I_{i_{0}}^{\prime \prime}$ ), we associate the weights $\alpha_{i}^{\prime}:=\alpha_{i}$ (resp. $\alpha_{i}^{\prime \prime}:=\sum \alpha_{k}$ where the sum runs over all the $H_{k}^{\prime} \in \mathcal{A}_{i 0}^{\prime}$ such that $H_{i}^{\prime \prime} \subset H_{k}^{\prime}$ ).

There is an inductive definition (deletion-restriction) of $\beta \mathrm{nbc}_{i_{0}}(\mathcal{A})$ : 
Proposition 2.4.8 (Ziegler[Z, Theorem 1.5] [FT, Theorem 2.4]) Let $\left(\mathcal{A}, \mathcal{A}_{i_{0}}^{\prime}, \mathcal{A}_{i_{0}}^{\prime \prime}\right)$ be the triple defined above. Suppose that $\mathcal{A}_{i_{0}}^{\prime}$ is essential. Then

$$
\beta \operatorname{nbc}_{i_{0}}(\mathcal{A})=\beta \operatorname{nbc}\left(\mathcal{A}_{i_{0}}^{\prime}\right) \cup\left\{\left(\nu\left(B^{\prime \prime}\right), H_{i_{0}}\right) \mid B^{\prime \prime} \in \beta \operatorname{nbc}\left(\mathcal{A}_{i_{0}}^{\prime \prime}\right)\right\}
$$

where $\nu\left(B^{\prime \prime}\right)=\left(\nu\left(H_{1}^{\prime \prime}\right), \cdots, \nu\left(H_{k}^{\prime \prime}\right)\right)$ if $B^{\prime \prime}=\left(H_{1}^{\prime \prime}, \cdots, H_{k}^{\prime \prime}\right)$.

Remark 2.4.9 Write $\overline{\beta \mathrm{nbc}}\left(\mathcal{A}_{i_{0}}^{\prime \prime}\right)=\left\{\left(\nu\left(B^{\prime \prime}\right), H_{i_{0}}\right) \mid B^{\prime \prime} \in \beta \operatorname{nbc}\left(\mathcal{A}_{i_{0}}^{\prime \prime}\right)\right\}$. Elements of $\beta \operatorname{nbc}\left(\mathcal{A}_{i_{0}}^{\prime}\right)$ are always less than elements of $\overline{\beta \mathrm{nbc}}\left(\mathcal{A}_{i_{0}}^{\prime \prime}\right)$.

Lemma 2.4.10 If $B \in \beta \operatorname{nbc}\left(\mathcal{A}_{i_{0}}^{\prime}\right)$, then $\Xi(B, \mathcal{A})_{\mid \alpha_{i_{0}}=0}=\Xi^{\prime}\left(B, \mathcal{A}_{i_{0}}^{\prime}\right)$.

Proof. Let $F \in L(\mathcal{A})$. If $i_{0} \notin I(F)$, then $\omega_{\alpha}(F, \mathcal{A})=\omega_{\alpha}\left(F, \mathcal{A}_{i_{0}}^{\prime}\right)$ and if $i_{0} \in I(F)$, then $\omega_{\alpha}(F, \mathcal{A})=\omega_{\alpha}\left(F, \mathcal{A}_{i_{0}}^{\prime}\right)+\psi \alpha_{i_{0}} \omega_{i_{0}}$ for some form $\psi$.

Lemma 2.4.11 Let $B^{\prime \prime} \in \beta \operatorname{nbc}\left(\mathcal{A}_{i_{0}}^{\prime \prime}\right)$ and $B=\left(\nu\left(B^{\prime \prime}\right), H_{i_{0}}\right) \in \overline{\beta \operatorname{nbc}}\left(\mathcal{A}_{i_{0}}^{\prime \prime}\right)$. Then the residue of $\Xi(B, \mathcal{A})$ along $H_{i_{0}}$ is equal to $\Xi\left(B^{\prime \prime}, \mathcal{A}_{i_{0}}^{\prime \prime}\right)$.

Proof. The last factor of $\Xi(B, \mathcal{A})$ is $\alpha_{i_{0}} \omega_{i_{0}}$. Since the product is the exterior one, it follows that $\alpha_{i_{0}} \omega_{i_{0}}$ may be removed as a summand from all the other factors of the product without changing its value. Taking residue of this rewritten product removes the factor $\alpha_{i_{0}} \omega_{i_{0}}$ and restricts the remaining terms to $H_{i_{0}}$. The residue is now just $\Xi\left(B^{\prime \prime}, \mathcal{A}_{i_{0}}^{\prime \prime}\right)$.

Example 2.4.12 Let $\mathcal{A}$ be an arrangement in general position. Consider the standard order $<_{p}$. Then $\beta(\mathcal{A})=\left(\begin{array}{c}p-1 \\ n\end{array}\right)$.

$$
\beta \operatorname{nbc}_{p}(\mathcal{A})=\left\{\left(H_{i_{1}}, \ldots, H_{i_{n}}\right) \mid 1<i_{1}<\cdots<i_{n} \leq p\right\}
$$

and

$$
\Phi^{p}(\mathcal{A})=\left\{\alpha_{i_{1}} \cdots \alpha_{i_{n}} \omega_{i_{1} \cdots i_{n}} \mid 1<i_{1}<\cdots<i_{n} \leq p\right\} .
$$

Example 2.4.13 Let $\mathcal{A}=\left\{H_{i}\right\}_{1 \leq i \leq 4}$ with

$$
f_{1}(x, y)=x-y, f_{2}(x, y)=1-x, f_{3}(x, y)=y, f_{4}(x, y)=1-y, f_{5}(x, y)=x .
$$

(i) Consider the order $<_{1}$ :

$$
H_{2}<_{1} H_{3}<_{1} H_{4}<_{1} H_{5}<_{1} H_{1} .
$$

- The circuits are $\left(H_{2}, H_{4}, H_{1}\right)$ and $\left(H_{3}, H_{5}, H_{1}\right)$ and the broken circuits are $\left(H_{4}, H_{1}\right)$ and $\left(H_{5}, H_{1}\right)$. Thus

$$
\operatorname{nbc}_{1}(\mathcal{A})=\left\{\left(H_{2}, H_{1}\right),\left(H_{3}, H_{1}\right),\left(H_{2}, H_{3}\right),\left(H_{2}, H_{4}\right),\left(H_{3}, H_{5}\right),\left(H_{4}, H_{5}\right)\right\}
$$


and

$$
\beta \operatorname{nbc}_{1}(\mathcal{A})=\left\{\left(H_{4}, H_{5}\right),\left(H_{3}, H_{1}\right)\right\}
$$

- We have

$$
\begin{gathered}
\mathcal{A}_{1}^{\prime}=\left\{H_{2}<H_{3}<H_{4}<H_{5}\right\}, \\
\mathcal{A}_{1}^{\prime \prime}=\left\{H_{2} \cap H_{1}, H_{3} \cap H_{1}\right\},
\end{gathered}
$$

$\beta \operatorname{nbc}\left(\mathcal{A}_{1}^{\prime}\right)=\left\{\left(H_{4}, H_{5}\right)\right\}, \beta \operatorname{nbc}\left(\mathcal{A}_{1}^{\prime \prime}\right)=\left\{H_{351}\right\}\left(H_{351}:=H_{3} \cap H_{5}=H_{3} \cap H_{5} \cap H_{1}, \nu\left(H_{351}\right)=\right.$ $\left.\min \left(H_{3}, H_{5}, H_{1}\right)=H_{3}\right)$ and

$$
\beta \operatorname{nbc}_{1}(\mathcal{A})=\beta \operatorname{nbc}\left(\mathcal{A}_{1}^{\prime \prime}\right) \cup \overline{\beta \operatorname{nbc}}\left(\mathcal{A}_{1}^{\prime \prime}\right)
$$

$$
\Phi^{1}=\left\{\alpha_{4} \alpha_{5} \omega_{45}, \alpha_{3} \alpha_{1} \omega_{31}+\alpha_{5} \alpha_{1} \omega_{51}\right\}
$$

(ii) In the same way,

$$
\begin{aligned}
& \Phi^{2}=\left\{\alpha_{4} \alpha_{5} \omega_{45}, \alpha_{3} \alpha_{2} \omega_{32}\right\} \\
& \Phi^{3}=\left\{\alpha_{4} \alpha_{5} \omega_{45}, \alpha_{2} \alpha_{3} \omega_{23}\right\} \\
& \Phi^{4}=\left\{\alpha_{2} \alpha_{3} \omega_{23}, \alpha_{5} \alpha_{4} \omega_{54}\right\} \\
& \Phi^{5}=\left\{\alpha_{2} \alpha_{3} \omega_{23}, \alpha_{4} \alpha_{5} \omega_{45}\right\}
\end{aligned}
$$

\section{Hypergeometric period matrix}

\subsection{The $\beta$ nbc-ordered homology basis}

Recall that $\operatorname{Ch}(\mathcal{A})$ is the set of real bounded chambers of $\mathcal{A}$. Let $\beta=\beta(\mathcal{A})=|\operatorname{Ch}(\mathcal{A})|$. In order to define the period matrix, we label $C h(\mathcal{A})$ by $\beta \mathrm{nbc}_{i_{0}}(\mathcal{A})$.

Definition 3.1.1 Let $\xi=\left(F_{0} \subset F_{1} \subset \cdots \subset F_{n}\right)$ be a flag of affine subspaces $F_{i} \in L(\mathcal{A})$ with $\operatorname{dim} F_{i}=i(i=0, \ldots, n)$. Let $\Delta \in \operatorname{Ch}(\mathcal{A})$ and $\bar{\Delta}$ be its closure in $\mathbb{R}^{n}$. We say that $\xi$ is adjacent to $\Delta$ if $\operatorname{dim}\left(F_{i} \cap \bar{\Delta}\right)=i$ for $i=0, \ldots, n$.

For $B=\left(H_{i_{1}}, \ldots, H_{i_{n}}\right) \in \beta \mathrm{nbc}_{i_{0}}(\mathcal{A})$, recall the associated $\beta$ nbc-flag $\xi(B)=\left(F_{0} \subset\right.$ $\left.F_{1} \subset \cdots \subset F_{n}\right)$ from Definition 2.4.4.

Proposition 3.1.2 There exists a unique bijection

$$
C: \beta \operatorname{nbc}_{i_{0}}(\mathcal{A}) \longrightarrow \mathrm{Ch}(\mathcal{A})
$$

with the property that $\xi(B)$ is adjacent to the bounded chamber $C(B)$ for any $B \in \beta \mathrm{nbc}_{i_{0}}(\mathcal{A})$. 
Proof. If $\beta \mathrm{nbc}_{i_{0}}(\mathcal{A})=\emptyset$, then $\mathrm{Ch}(\mathcal{A})=\emptyset$. Suppose $\beta \mathrm{nbc}_{i_{0}}(\mathcal{A}) \neq \emptyset$. We will prove by induction on $|\mathcal{A}|$. Assume that the maps $C^{\prime}$ and $C^{\prime \prime}$ already exist for $\mathcal{A}_{i_{0}}^{\prime}$ and $\mathcal{A}_{i_{0}}^{\prime \prime}$. There are the following four kinds of bounded chambers of $\mathcal{A}$ :

(i) $\Delta \in \mathrm{Ch}(\mathcal{A})$ is called undivided if $\Delta \in \mathrm{Ch}\left(\mathcal{A}_{i_{0}}^{\prime}\right)$, i.e., $\Delta$ does not intersect $H_{i_{0}}$.

(ii) $\Delta \in \mathrm{Ch}(\mathcal{A})$ is called newborn if there exists an unbounded chamber of $\mathcal{A}_{i_{0}}^{\prime}$ which contains $\Delta$.

(iii) Suppose that a bounded chamber $\Delta^{\prime}$ of $\mathcal{A}_{i_{0}}^{\prime}$ is divided in two by $H_{i_{0}}$. (In this case, a bounded chamber $\Delta^{\prime}$ of $\mathcal{A}_{i_{0}}^{\prime}$ is called divided.) Let $B^{\prime} \in \beta \operatorname{nbc}\left(\mathcal{A}_{i_{0}}^{\prime}\right)$ with $\Delta^{\prime}=C^{\prime}\left(B^{\prime}\right)$. Then $\xi^{\prime}:=\xi\left(B^{\prime}\right)$ is adjacent to $\Delta^{\prime}$. The two new chambers of $\mathcal{A}$ inside $\Delta^{\prime}$ are denoted by $\Delta^{+}$and $\Delta^{-}$. We can easily observe that $\xi^{\prime}$ is adjacent to exactly one of the two chambers $\Delta^{+}$and $\Delta^{-}$, say, $\Delta^{+}$. The chamber $\Delta^{+} \in \operatorname{Ch}(\mathcal{A})$ is called the heir of $\Delta^{\prime}$. The other chamber $\Delta^{-} \in \mathrm{Ch}(\mathcal{A})$ is called the cutoff of $\Delta^{\prime}$.

Define $C: \beta \mathrm{nbc}_{i_{0}}(\mathcal{A}) \longrightarrow \mathrm{Ch}(\mathcal{A})$ as follows:

(a) If $B \in \beta \operatorname{nbc}\left(\mathcal{A}_{i_{0}}^{\prime}\right)$ and $C^{\prime}(B) \in \mathrm{Ch}(\mathcal{A})$, then let $C(B)=C^{\prime}(B)$.

(b) If $B \in \beta \operatorname{nbc}\left(\mathcal{A}_{i_{0}}^{\prime}\right)$ and $C^{\prime}(B)$ is divided in two by $H_{i_{0}}$, then define $C(B)=\Delta^{+}$, where $\Delta^{+}$is the heir of $C^{\prime}(B)$.

(c) If $B=\left(\nu B^{\prime \prime}, H_{i_{0}}\right)$ with $B^{\prime \prime} \in \beta \mathrm{nbc}\left(\mathcal{A}_{i_{0}}^{\prime \prime}\right)$ and $C^{\prime \prime}\left(B^{\prime \prime}\right)$ is inside an unbounded chamber of $\mathcal{A}_{i 0}^{\prime}$, then $C^{\prime \prime}\left(B^{\prime \prime}\right)$ is a wall of a unique newborn chamber $\Delta$. Define $C(B)=\Delta$.

(d) If $B=\left(\nu B^{\prime \prime}, H_{i_{0}}\right)$ with $B^{\prime \prime} \in \beta \operatorname{nbc}\left(\mathcal{A}_{i_{0}}^{\prime \prime}\right)$ and $C^{\prime \prime}\left(B^{\prime \prime}\right)$ is inside a bounded chamber $\Delta^{\prime}$ of $\mathcal{A}_{i_{0}}^{\prime}$, then $C^{\prime \prime}\left(B^{\prime \prime}\right)$ is a wall of a unique cutoff chamber $\Delta^{-}$of $\Delta^{\prime}$. Define $C(B)=\Delta^{-}$.

By construction, $C$ is bijective and satisfies the condition. The uniqueness is obvious from the construction.

Let $\beta \mathrm{nbc}_{i_{0}}(\mathcal{A})=\left\{B_{1}, \ldots, B_{\beta}\right\}$ be linearly ordered as in section 2.4. Define $\Delta_{i}:=$ $C\left(B_{i}\right) \in \operatorname{Ch}(\mathcal{A})$ for $i=1, \ldots, \beta$. We call $\Delta_{1}, \ldots, \Delta_{\beta}$ the $\beta$ nbc $_{i_{0}}$-ordered chambers of $\mathcal{A}$. If $\Delta_{i}$ is either undivided or an heir and $\Delta_{j}$ is either newborn or a cutoff, then $i<j$.

We give an orientation to each $\Delta \in C h(\mathcal{A})$ as follows: Let $\Delta=C(B)$ with $B \epsilon$ $\beta \mathrm{nbc}_{i_{0}}(\mathcal{A})$. Let $\xi(B)=\left(F_{0} \subset F_{1} \subset \cdots \subset F_{n}\right)$ be the associated $\beta$ nbc-flag. Choose the intrinsic orientation [V2, 6.2] of $\Delta$ obtained from $\xi(B)$. In other words, an orthonormal frame $\left\{e_{1}, \ldots, e_{n}\right\}$ is chosen so that each $e_{i}$ is a unit vector originating from the point $F_{0}$ in the direction of $F_{i} \cap \bar{\Delta}$. This orientation is called the $\beta$ nbc-orientation of $\Delta$.

Example 3.1.3 Recall Example 2.4.13 with the order $<_{1}$ :

$$
H_{2}<_{1} H_{3}<_{1} H_{4}<_{1} H_{5}<_{1} H_{1} .
$$

Let $B_{1}=\left(H_{4}, H_{5}\right)$ and $B_{2}=\left(H_{3}, H_{1}\right)$. Then $\beta \mathrm{nbc}_{1}(\mathcal{A})=\left\{B_{1}, B_{2}\right\}$. 


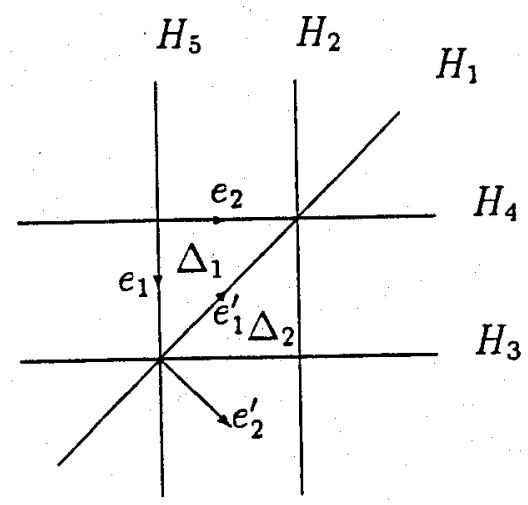

The bijection map

$$
C: \beta \mathrm{nbc}_{3}(\mathcal{A}) \longrightarrow \mathrm{Ch}(\mathcal{A})
$$

described in Proposition 3.1.2 is given by $C\left(B_{i}\right)=\Delta_{i}$ for $i=1,2$. The $\beta$ nbc-flags are

$$
\xi\left(B_{1}\right)=\left(H_{4} \cap H_{5} \subset H_{5} \subset V\right)
$$

and

$$
\xi\left(B_{2}\right)=\left(H_{3} \cap H_{1} \subset H_{1} \subset V\right) .
$$

The $\beta$ nbc-orientations of $\Delta_{1}$ and $\Delta_{2}$ are given by orthonormal frames $\left\{e_{1}, e_{2}\right\}$ and $\left\{e_{1}^{\prime}, e_{2}^{\prime}\right\}$ respectively.

Let $\mathcal{L}_{\alpha}$ be the rank one local system on $M=M(\mathcal{A})$ (cf. 2.1.4) defined by the kernel of an integral connection

$$
\nabla_{\alpha}: \mathcal{O}_{M} \longrightarrow \Omega_{M}^{1}
$$

where $\nabla_{\alpha}(f)=d f+f \sum_{i \in I} \alpha_{i} \omega_{i}$. Then its monodromy around $H_{k}$ is $e^{-2 i \pi \alpha_{k}}$.

Proposition 3.1.4 (cf. [Ko], [AK, 4.1.1]) Suppose that the weights $\alpha=\left(\alpha_{1}, \cdots, \alpha_{p}\right)$ are non-resonant (cf. Definition 2.3.2). We have

(i) $H_{j}\left(M(\mathcal{A}), \mathcal{L}_{\alpha}\right)=H_{j}^{l f}\left(M(\mathcal{A}), \mathcal{L}_{\alpha}\right)=0$ pour $j \neq n$. Here $H^{l f}$ stands for the locally finite homology.

(ii) The natural map

$$
H_{n}\left(M(\mathcal{A}), \mathcal{L}_{\alpha}\right) \longrightarrow H_{n}^{l f}\left(M(\mathcal{A}), \mathcal{L}_{\alpha}\right)
$$

is an isomorphism.

(iii) $\left\{\left[\Delta_{j}\right] \mid \Delta_{j} \in \mathrm{Ch}(\mathcal{A})\right\}$ forms a basis for $H_{n}^{l f}\left(M(\mathcal{A}), \mathcal{L}_{\alpha}\right)$.

Proof. The following proof is similar to Kohno's proof [Ko] in the case that the arrangement is generic to infinity.

For (i) and (ii), we use the exactly same argument as [Ko, Theorem 1] except that we blow up $\mathbb{P}^{n}$ along all the dense edges of codimension of codimension $>1$. 
(iii): Write $M=M(\mathcal{A})$ and $\Delta=\bigcup_{j=1}^{\beta} \Delta_{j}$. In order to apply the argument in [Ko], it is enough to show $H^{q}\left(M-\Delta, \mathcal{L}_{\alpha}\right)=0$ for all $q$. Let $W$ be a small tubular neighborhood in $\mathbb{P}^{n}$ of the hyperplane at infinity $\bar{H}_{\infty}$. Note that the inclusion map $W \cap M \hookrightarrow M-\Delta$ is homotopy equivalent.

Let $j: W \cap M \hookrightarrow W$. Let $x \in W$. If $x \in W \cap M$, since there is no hyperplane in $\mathcal{A}_{\infty}$ going through $x$ we have $\left(R^{q} j_{x} \mathcal{L}_{\alpha}\right)_{x}=0$ for $q \neq 0$ and $\left(R^{0} j_{*} \mathcal{L}_{\alpha}\right)_{x} \simeq \mathbb{C}$. If $x \in W-M$, then $W-M$ is locally a central arrangement near $x$. In this case, since the (local) Euler characteristic of $M$ (intersected with a small open ball centered at $x)$ is zero, we have $\left(R^{q} j_{x} \mathcal{L}_{\alpha}\right)_{x}=0$ for all $q$ by (i). Therefore we have $H^{q}\left(W \cap M, \mathcal{L}_{\alpha}\right) \simeq H^{q}\left(W, j_{=} \mathcal{L}_{\alpha}\right)$. For each $x \in \bar{H}_{\infty}$, there exists a small neighborhood $W_{x}$ of $x$ in $W$ such that

$$
H^{q}\left(W_{x_{1}} \cap \cdots \cap W_{x_{k}}, j_{x} \mathcal{L}_{\alpha}\right)=0
$$

as long as $W_{x_{1}} \cap \cdots \cap W_{x_{k}} \neq \emptyset$. Since $\bar{H}_{\infty} \simeq \mathbb{P}^{n-1}$ is compact, we may choose $W_{x_{1}}, \ldots, W_{x_{m}}$ which cover $\bar{H}_{\infty}$. Let $W_{0}=W_{x_{1}} \cup \cdots \cup W_{x_{m}}$. By applying the Mayer-Vietoris theorem repeatedly, we have $H^{q}\left(W_{0}, j_{2} \mathcal{L}_{\alpha}\right)=0$ for all $q$. By the Poincare duality, we have

$$
H^{q}\left(M-\Delta, \mathcal{L}_{\alpha}\right) \simeq H^{q}\left(W_{0} \cap M, \mathcal{L}_{\alpha}\right) \simeq H^{q}\left(W_{0}^{\dagger}, j_{*} \mathcal{L}_{\alpha}\right)=0
$$

\subsection{The $\beta$ nbc-ordered cohomology basis}

Proposition 3.2.1 Suppose that the weights $\alpha=\left(\alpha_{1}, \cdots, \alpha_{p}\right)$ are non-resonant. We have

(i) $H^{j}\left(M(\mathcal{A}), \mathcal{L}_{\alpha}\right)=H_{c}^{j}\left(M(\mathcal{A}), \mathcal{L}_{\alpha}\right)=0$ pour $j \neq n$. Here $H_{c}$ stands for the compact support cohomology.

(ii) The natural map

$$
H_{c}^{n}\left(M(\mathcal{A}), \mathcal{L}_{\alpha}\right) \longrightarrow H^{n}\left(M(\mathcal{A}), \mathcal{L}_{\alpha}\right)
$$

is an isomorphism.

(iii) The set $\Phi^{i_{0}}(\mathcal{A})$ (cf. Definition 2.4.6) forms a basis for $H^{n}\left(M(\mathcal{A}), \mathcal{L}_{\alpha}\right)$.

Proof. Let $\mathcal{L}_{\alpha}^{\vee}$ be the dual local system of $\mathcal{L}_{\alpha}$. (i) and (ii) are obtained from the Poincaré dualities

$$
H^{q}\left(M(\mathcal{A}), \mathcal{L}_{\alpha}\right) \simeq H_{2 n-q}^{l f}\left(M(\mathcal{A}), \mathcal{L}_{\alpha}^{\vee}\right), H_{c}^{q}\left(M(\mathcal{A}), \mathcal{L}_{\alpha}\right) \simeq H_{2 n-q}\left(M(\mathcal{A}), \mathcal{L}_{\alpha}^{\vee}\right)
$$

and Proposition 3.1.4. (iii) is [FT, Theorem 3.7].

If $j_{0} \in I$ such that $j_{0} \neq i_{0}$, it may happens that $\Phi^{j_{0}} \neq \Phi^{i_{0}}$ (cf. Example 2.4.13). However we have the following

Proposition 3.2.2 [FT, Proposition 3.10] For all $j_{0} \in I$ the transition matrix between the bases $\Phi^{i_{0}}$ and $\Phi^{j_{0}}$ is an integral unimodular matrix independent of $\alpha$. 


\subsection{The definition of hypergeometric period matrix}

Definition 3.3.1 Let $\beta=\beta(\mathcal{A})$. Assume that $\mathrm{Ch}(\mathcal{A})=\left\{\Delta_{1}, \ldots, \Delta_{\beta}\right\}$ is the $\beta$ nbc-ordered chambers in 3.1.4 (iii) and $\Phi^{i_{0}}(\mathcal{A})=\left\{\phi_{1}^{i_{0}}, \ldots, \phi_{\beta}^{i_{0}}\right\}$ is the $\beta$ nbc-ordered basis of $H^{n}\left(M, \mathcal{L}_{\alpha}\right)$ in 3.2.1 (iii). Choose a branch of $f_{j}^{\alpha_{j}}$ on each chamber $\Delta_{i}$. Let

$$
U_{\alpha}:=f_{1}^{\alpha_{1}} \cdots f_{p}^{\alpha_{p}} \text {. }
$$

Also choose the $\beta$ nbc-orientation of each chamber $\Delta_{i}$. Define the hypergeometric period matrix $\mathrm{PM}_{i_{0}}(\mathcal{A}, \alpha)$ by

$$
\mathrm{PM}_{i_{0}}(\mathcal{A}, \alpha)=\left[\int_{\Delta,} U_{\alpha} \phi_{i}^{i_{0}}\right]
$$

If $\Re \alpha_{j}>0, \forall j \in I$, then each entry of $\mathrm{PM}_{i_{0}}(\mathcal{A}, \alpha)$ can be regarded as an holomorphic function of $\alpha=\left(\alpha_{1}, \cdots, \alpha_{p}\right)$ and it can be analytically continued to be a meromorphic function on the entire $\mathbb{C}^{p}$. In order to precise this point, first introduce the following Definition 3.3.2 Let $\Delta_{j}^{*}$ be a twisted cycle representing the homology class in $H_{n}\left(M(\mathcal{A}), \mathcal{L}_{\alpha}^{\vee}\right)$ which is sent to the homology class of $\Delta_{j}$ in $H_{n}^{l f}\left(M(\mathcal{A}), \mathcal{L}_{\alpha}^{\vee}\right)$ via the isomorphism in Proposition 3.1.4 (ii). Define

$$
\mathrm{PM}_{i_{0}}^{*}(\mathcal{A}, \alpha)=\left[\int_{\Delta ;} U_{\alpha} \phi_{i}^{i_{0}}\right]
$$

Remark 3.3.3 It is known (e.g., see [LS, 4.2]) that each entry of $\mathrm{PM}_{i_{0}}^{*}(\mathcal{A}, \alpha)$ can be regarded as a meromorphic function on $\mathbb{C}^{p}$ whose poles lie on some hypersurfaces defined by equations $e^{2 i \pi L(\alpha)}-\lambda=0$, where $L$ is a linear form of $\alpha_{1}, \cdots, \alpha_{p}$ and $\lambda$ is a nonzero complex number. Since the twisted de Rham pairing

$$
H_{n}\left(M(\mathcal{A}), \mathcal{L}_{\alpha}^{\vee}\right) \times H^{n}\left(M(\mathcal{A}), \mathcal{L}_{\alpha}\right) \longrightarrow \mathbb{C}
$$

which is given by the hypergeometric integrals $\left(\Delta^{*}, \phi\right) \mapsto \int_{\Delta} . U_{\alpha} \phi$, is a nondegenerate pairing (e.g., see [Ki, 1.4]), we may write $L(\alpha)=\alpha(F)$ for a dense edge $F$ and $\lambda=1$ by Propositions 3.1.4 (iii) and 3.2.1 (iii). In other words, $\operatorname{det} \operatorname{PM}_{i_{0}}^{-}(\mathcal{A}, \alpha)$ takes a finite nonzero value at each $\alpha \notin \operatorname{Rsn}(\mathcal{A})$. Moreover, if $\Re \alpha_{j}>0, \forall j \in I$, then

$$
\int_{\Delta ;} U_{\alpha} \phi_{i}^{i_{0}}=\int_{\Delta,} U_{\alpha} \phi_{i}^{i_{0}}
$$

In particular, if $\Re \alpha_{j}>0$ for all $j \in I$,

$$
\mathrm{PM}_{i_{0}}^{*}(\mathcal{A}, \alpha)=\mathrm{PM}_{i_{0}}(\mathcal{A}, \alpha)
$$

and the analytic continuation of the determinant of $\mathrm{PM}_{i_{0}}(\mathcal{A}, \alpha)$ is equal to the determinant of $\mathrm{PM}_{i_{0}}^{*}(\mathcal{A}, \alpha)$.

Remark 3.3.4 We formally define $\operatorname{det} \mathrm{PM}_{i_{0}}(\mathcal{A}, \alpha)=1$ if $\beta(\mathcal{A})=0$.

Proposition 3.3.5 The determinant of $\operatorname{PM}_{i_{0}}(\mathcal{A}, \alpha)$ is independent (up to sign) of the choice of $i_{0}$.

Proof. Obvious from Proposition 3.2.2. 


\section{Beta function of an arrangement}

We keep the notations of Section 2 .

\section{1}

Let $\mathcal{A}$ be an affine arrangement and $\mathcal{A}_{\infty}$ the arrangement of projective hyperplanes defined in 2.3. Recall $\bar{I}=I \cup\{\infty\}=\{1, \ldots, p, \infty\}$ and $\alpha_{\infty}=-\left(\alpha_{1}+\cdots+\alpha_{p}\right)$. We note that $L_{-}\left(\mathcal{A}_{\infty}\right)\left(\right.$ or $\left.L_{+}\left(\mathcal{A}_{\infty}\right)\right)$ is the set of edges of $L\left(\mathcal{A}_{\infty}\right)$ contained (resp. not contained) in $\bar{H}_{\infty}$. For $F \in L\left(\mathcal{A}_{\infty}\right)$, let

$$
\begin{gathered}
I(F)=\left\{i \in I \mid F \subset H_{i}\right\}, \\
\bar{I}(F)=\left\{i \in \bar{I} \mid F \subset \bar{H}_{i}\right\}, \\
\mathcal{A}_{\infty}^{F}=\left\{\bar{H}_{i} \mid i \in \bar{I}(F)\right\}, \\
\mathcal{A}_{F}^{\infty}=\left\{\bar{H}_{i} \cap F \mid F \nsubseteq \bar{H}_{i}\right\}, \\
\alpha(F)=\sum_{i \in \bar{I}(F)} \alpha_{i}= \begin{cases}\sum_{i \in I(F)} \alpha_{i} & \text { if } F \in L_{+}\left(\mathcal{A}_{\infty}\right), \\
-\sum_{i \in I-\bar{I}(F)} \alpha_{i} & \text { if } F \in L_{-}\left(\mathcal{A}_{\infty}\right) .\end{cases}
\end{gathered}
$$

Following Varchenko $[\mathrm{V} 1,1.5]$, we associate to each edge $F$ of codimension $r$ a projective arrangement $\mathbb{P} \mathcal{A}_{\infty}^{F}$ in the $r-1$ dimensional projective space. If $\mathcal{A}_{\infty}$ is a projective arrangement, let $\chi\left(\mathcal{A}_{\infty}\right)$ denote the Euler characteristic of $\mathbb{P}^{n}-\cup_{i \in \bar{I}} \bar{H}_{i}$. If $F$ is an edge of $\mathcal{A}_{\infty}$ defined above, we put

$$
\mu\left(F, \mathcal{A}_{\infty}\right)=\left|\chi\left(\mathcal{A}_{F}^{\infty}\right) \chi\left(\mathbb{P} \mathcal{A}_{\infty}^{F}\right)\right|
$$

Proposition 4.1.1 If an edge $F \in L\left(\mathcal{A}_{\infty}\right)$ is not dense, then $\mu\left(F, \mathcal{A}_{\infty}\right)=0$.

Proof. Recall that $F$ is dense if and only if $\chi\left(\mathbb{P} \mathcal{A}_{\infty}^{F}\right) \neq 0$ [STV, Proposition 7].

Definition 4.1.2 Let $j \in I$ and $i \in\{1, \ldots, \beta(\mathcal{A})\}$. Choose a branch of $f_{j}^{\alpha}$ on each $\Delta_{i}$. Define

$$
\begin{gathered}
R\left(f_{j}, \Delta_{i}\right)^{\alpha_{j}}=\left\{f_{j}^{\alpha_{j}}(x)|| f_{j}^{\alpha_{j}}(x)|\geq| f_{j}^{\alpha_{j}}(y) \mid, \forall y \in \overline{\Delta_{i}}\right\} \\
R(\mathcal{A})^{\alpha}=\prod_{j=1}^{p} \prod_{i=1}^{\beta} R\left(f_{j}, \Delta_{i}\right)^{\alpha_{j}}
\end{gathered}
$$

and

$$
B(\mathcal{A}, \alpha)=\prod_{F \in L_{+}\left(\mathcal{A}_{\infty}\right)} \Gamma(\alpha(F)+1)^{\mu\left(F, \mathcal{A}_{\infty}\right)} \prod_{F \in L_{-}\left(\mathcal{A}_{\infty}\right)} \Gamma(-\alpha(F)+1)^{-\mu\left(F, \mathcal{A}_{\infty}\right)} .
$$

Remark 4.1.3 Define $B(\mathcal{A}, \alpha)=1$ and $R(\mathcal{A})^{\alpha}=1$ if $\beta(\mathcal{A})=0$.

Remark 4.1.4 By Proposition 4.1.1 $B(\mathcal{A}, \alpha)$ involves only with $\alpha(F)$ for dense edges $F$. 


\subsection{Recursion formulas for $B(\mathcal{A}, \alpha)$ and $R(\mathcal{A})^{\alpha}$.}

Let $i_{0} \in I$. Define the order $<_{i_{0}}$ in $\mathcal{A}$, and orders in $\mathcal{A}_{i_{0}}^{\prime}$ and $\mathcal{A}_{i_{0}}^{\prime \prime}$ as in 2.4.7. Define

$$
I_{i_{0}}^{*}=\left\{i \in I_{i_{0}}^{\prime} \mid H_{i} \cap H_{i_{0}}=\emptyset\right\} \text {. }
$$

Theorem 4.2.1 We have

$$
\begin{aligned}
B(\mathcal{A}, \alpha)= & B\left(\mathcal{A}_{i_{0}}^{\prime}, \alpha^{\prime}\right) B\left(\mathcal{A}_{i_{0}}^{\prime \prime}, \alpha^{\prime \prime}\right) \\
& \times \prod_{F \in L_{+}\left(\mathcal{A}_{\infty}\right), F \subseteq H_{i_{0}}}\left[\Gamma(\alpha(F)+1) / \Gamma\left(\alpha(F)-\alpha_{i_{0}}+1\right)\right]^{\mu\left(F, \mathcal{A}_{\infty}\right)} \\
& \times \prod_{F \in L_{-}\left(\mathcal{A}_{\infty}\right), F \notin H_{i_{0}}}\left[\Gamma\left(-\alpha(F)-\alpha_{i_{0}}+1\right) / \Gamma(-\alpha(F)+1)\right]^{\mu\left(F, \mathcal{A}_{\infty}\right)}
\end{aligned}
$$

and

$$
R(\mathcal{A})^{\alpha}=R\left(\mathcal{A}_{i_{0}}^{\prime}\right)^{\alpha^{\prime}} R\left(\mathcal{A}_{i_{0}}^{\prime \prime}\right)^{\alpha^{\prime \prime}} \prod_{i=1}^{\mu} R\left(f_{i_{0}}, \Delta_{i}\right)^{\alpha_{i_{0}}}\left[\left.\prod_{i \in I_{i_{0}}^{\prime}} f_{i}^{\alpha_{i}}\right|_{H_{i_{0}}}\right]^{\beta\left(\mathcal{A}_{i_{0}}^{\prime \prime}\right)}
$$

Proof. Analogous to the proof of Proposition 6.3 of [L].

Corollary 4.2.2 Assume the arrangement $\mathcal{A}$ normal. Then

$$
\begin{gathered}
B(\mathcal{A}, \alpha)=B\left(\mathcal{A}_{i_{0}}^{\prime}, \alpha^{\prime}\right) B\left(\mathcal{A}_{i_{0}}^{\prime \prime}, \alpha^{\prime \prime}\right) \Gamma\left(\alpha_{i_{0}}+1\right)^{\beta\left(\mathcal{A}_{i_{0}}^{\prime \prime}\right)} \\
\times \prod_{F \in L_{-}\left(\mathcal{A}_{\infty}\right), F \nsubseteq H_{i_{0}}}\left[\Gamma\left(-\alpha(F)-\alpha_{i_{0}}+1\right) / \Gamma(-\alpha(F)+1)\right]^{\mu\left(F, \mathcal{A}_{\infty}\right)}
\end{gathered}
$$

This result was proved first by Varchenko ([V1, Theorem 2.5]).

Corollary 4.2.3 We have

$$
\left.B(\mathcal{A}, \alpha)\right|_{\alpha_{i_{0}}=0}=B\left(\mathcal{A}_{i_{0}}^{\prime}, \alpha^{\prime}\right) B\left(\mathcal{A}_{i_{0}}^{\prime \prime}, \alpha^{\prime \prime}\right)
$$

and

$$
\left.R(\mathcal{A})^{\alpha}\right|_{\alpha_{i_{0}}=0}=R\left(\mathcal{A}_{i_{0}}^{\prime}\right)^{\alpha^{\prime}} R\left(\mathcal{A}_{i_{0}}^{\prime \prime}\right)^{\alpha^{\prime \prime}}\left[\left.\prod_{j \in I_{i_{0}}^{*}} f_{j}^{\alpha}\right|_{H_{i_{0}}}\right]^{\beta\left(\mathcal{A}_{i_{0}}^{\prime \prime}\right)}
$$

Example 4.2.4 1. If $\mathcal{A}$ is in general position

$$
B(\mathcal{A}, \alpha)=\left[\prod_{i=1}^{p} \Gamma\left(\alpha_{i}+1\right) / \Gamma\left(\sum_{i=1}^{p} \alpha_{i}+1\right)\right]^{\left(\begin{array}{c}
p-2 \\
n-1
\end{array}\right)} .
$$

2. If $\mathcal{A}$ is the arrangement defined in Example 2.4.13,

$$
\begin{aligned}
B(\mathcal{A}, \alpha)= & \prod_{i=1}^{5} \Gamma\left(\alpha_{i}+1\right) \Gamma\left(\alpha_{1}+\alpha_{3}+\alpha_{5}+1\right) \Gamma\left(\alpha_{1}+\alpha_{2}+\alpha_{4}+1\right) \\
& \times\left[\Gamma\left(\sum_{i=1}^{5} \alpha_{i}+1\right) \Gamma\left(\alpha_{1}+\alpha_{3}+\alpha_{4}+1\right) \Gamma\left(\alpha_{1}+\alpha_{2}+\alpha_{5}+1\right)\right]^{-1}
\end{aligned}
$$




\section{The main theorem and its proof}

\subsection{The main theorem}

The main result of this paper is the following

Theorem 5.1.1 Suppose $\Re \alpha_{i}>0$ for all $i \in I$. Then, for all $i_{0} \in I$, we have

$$
\operatorname{det} \mathrm{PM}_{i_{0}}(\mathcal{A}, \alpha)=R(\mathcal{A})^{\alpha} B(\mathcal{A}, \alpha),
$$

$\mathcal{A}$ being equipped with the order $<_{i_{0}}$ (cf. 2.2.1).

Remark 5.1.2 $R(\mathcal{A})^{\alpha} B(\mathcal{A}, \alpha)$ is independent of $i_{0}$. Thus so is the determinant.

Let $B=\left(H_{i_{1}}, \ldots, H_{i_{n}}\right) \in \beta \mathrm{nbc}_{i_{0}}(\mathcal{A})$. Recall the associated $\beta$ nbc-flag

$$
\xi(B)=\left(F_{0} \subset F_{1} \subset \cdots \subset F_{n}\right) .
$$

Let $\beta \mathrm{nbc}_{i_{0}}(\mathcal{A})=\left\{B_{1}, B_{2}, \ldots, B_{\beta}\right\}$. It is shown [BV] that the set $\left\{\xi\left(B_{1}\right), \ldots, \xi\left(B_{\beta}\right)\right\}$ of the $\beta$ nbc-flags gives a $\mathbb{Z}$-basis for the flag complex cohomology $H^{n}(\mathcal{F})$ which is studied by Schechtman and Varchenko in [SV, sections 2, 3] [V3, 10.1]. (It is also known that $H^{n}(\mathcal{F})$ is naturally isomorphic to the reduced cohomology $\tilde{H}^{n-1}\left(K^{\prime}(\hat{L}), \mathbb{Z}\right)$ where $\hat{L}=L(\mathcal{A})-\{V\}$ and $K(\hat{L})$ is the order complex of $\hat{L}$ [FT, Remark 3.8].) For an arbitrary flag $\xi=\left(F_{0} \subset\right.$ $\left.F_{1} \subset \cdots \subset F_{n}\right), F_{i} \in L(\mathcal{A}), \operatorname{dim} F_{i}=i(i=0, \ldots, n)$, associate a differential $n$-form

$$
\Xi(\xi)=\omega_{\alpha}\left(F_{0}, \mathcal{A}\right) \wedge \cdots \wedge \omega_{\alpha}\left(F_{n-1}, \mathcal{A}\right)
$$

(cf. Notation 2.4.5). Consider the homomorphism

$$
\pi_{\alpha}: H^{n}(\mathcal{F}) \otimes \mathbb{C} \longrightarrow H^{n}\left(M(\mathcal{A}), \mathcal{L}_{\alpha}\right)
$$

such that $\pi_{\alpha}([\xi] \otimes 1)=[\Xi(\xi)]$. Then $\pi_{\alpha}\left(\left[\xi\left(B_{i}\right)\right] \otimes 1\right)=\left[\phi_{i}^{i_{0}}(\mathcal{A})\right]$ for $1 \leq i \leq \beta$. The map $\pi_{\alpha}$ is an isomorphism when $\alpha$ is non-resonant.

Corollary 5.1.3 Suppose $\Re \alpha_{i}>0$ for all $i \in I$. Choose a branch of $f_{j}^{\alpha}$ on each $\Delta_{i} \in \operatorname{Ch}(\mathcal{A})$. Let $\xi_{1}, \ldots, \xi_{\beta}$ be fiags of length $n+1$ such that their cohomology classes in the flag complex cohomology $H^{n}(\mathcal{F})$ form a $\mathbb{Z}$-basis. Let $\psi_{i}(\alpha)=\Xi\left(\xi_{i}\right)$ for $1 \leq i \leq \beta$. Then we have

$$
\operatorname{det}\left[\int_{\Delta_{j}} U_{\alpha} \psi_{i}(\alpha)\right]= \pm R(\mathcal{A})^{\alpha} B(\mathcal{A}, \alpha)
$$

Proof. Since $\left\{\xi\left(B_{1}\right), \ldots, \xi\left(B_{\beta}\right)\right\}$ and $\left\{\xi_{1}, \ldots, \xi_{\beta}\right\}$ are connected by a unimodular integral constant matrix in $H^{n}(\mathcal{F})$, so are $\Phi^{i_{0}}(\mathcal{A})$ and $\left\{\psi_{1}(\alpha), \ldots, \psi_{\beta}(\alpha)\right\}$ in $H^{n}\left(M(\mathcal{A}), \mathcal{L}_{\alpha}\right)$. Apply Theorem 5.1.1. 
Remark 5.1.4 Theorem 5.1.1 shows that the conjecture by Varchenko ([V2, 6.3 Fundamental conjecture]) is true for the $\beta$ nbc-bases and the $\beta$ nbc-orientations. If Varchenko's flags $F_{\Delta_{j}}(1 \leq j \leq \beta)$ in [V2,6.2] form a $\mathbb{Z}$-basis for $H^{n}(\mathcal{F})$, then the affirmative answer (up to sign) to the original conjecture follows from Corollary 5.1.3. (In general, the original conjecture by Varchenko is always true up to a constant integral multiple by Theorem 5.1.1. Especially, for 2-dimensional arrangements, $M$. Neergaard and the second author have verified the original conjecture by studying the relationship between the $\beta$ nbc-flags and Varchenko's flags.) Note that the basis $\Phi^{p}$ coincides (up to sign) with Varchenko's basis $\left\{\Xi\left(F_{\Delta_{j}}\right)\right\}$ when $\mathcal{A}$ is normal or in general position at infinity.

\subsection{A theorem by Loeser-Sabbah}

Recall, at first, one of the main results of [LS].

Theorem 5.2.1 We have

$$
\operatorname{det} \mathrm{PM}_{i_{0}}^{=}(\mathcal{A}, \alpha)=c_{1}^{\alpha_{1}} \ldots c_{p}^{\alpha_{p}} B(\mathcal{A}, \alpha) h_{i_{0}}(\alpha)
$$

where $c_{1}, \cdots, c_{p}$ are nonzero constants and $h_{i_{0}} \in \mathbb{C}\left(\alpha_{1}, \cdots, \alpha_{p}\right)^{\approx}$.

Proof. By [LS, 4.2.10], we have

$$
\operatorname{det} \mathrm{PM}_{i_{0}}^{*}(\mathcal{A}, \alpha)=\varphi_{i_{0}}\left(e^{2 i \pi \alpha_{1}}, \cdots, e^{2 i \pi \alpha_{p}}\right) c_{1}^{\alpha_{1}} \ldots c_{p}^{\alpha_{p}} B(\mathcal{A}, \alpha) \hat{h}_{i_{0}}(\alpha)
$$

where $\varphi_{i_{0}}$ is a periodic function of $\alpha=\left(\alpha_{1}, \cdots, \alpha_{p}\right), c_{1}, \cdots, c_{p}$ are nonzero constants and $\hat{h}_{i_{0}} \in \mathbb{C}\left(\alpha_{1}, \cdots, \alpha_{p}\right)^{*}$. Since the polynomials $f_{i}$ take real values on each $\Delta_{i}$ and $\operatorname{det} \operatorname{PM}_{i_{0}}^{*}(\mathcal{A}, \alpha)$ is holomorphic if $\Re \alpha_{i}>0$ for all $i \in I$ by Remark $3.3 .3, \varphi_{i_{0}}\left(e^{2 i \pi \alpha_{1}}, \cdots, e^{2 i \pi \alpha_{p}}\right)$ is constant by [LS, final remark] (see also Remark 5.4.3). Denote $\varphi_{i_{0}} \hat{h}_{i_{0}}(\alpha)$ by $h_{i_{0}}(\alpha)$.

Remark 5.2.2 The constants $c_{k}$ are defined as critical values (counted with multiplicities) of the polynomials $f_{k}$.

Recall that $\operatorname{det} \mathrm{PM}_{i_{0}}^{*}(\mathcal{A}, \alpha)=\operatorname{det} \mathrm{PM}_{i_{0}}(\mathcal{A}, \alpha)$ if $\Re \alpha_{i}>0$ for all $i \in I$. We shall show that $h_{i_{0}}$ equals +1 and $c_{k}^{\alpha_{k}}=\prod_{j=1}^{\beta} R\left(f_{k}, \Delta_{j}\right)^{\alpha_{k}}$.

\subsection{A recursion formula for hypergeometric period determinants}

Let $i_{0} \in I$. Give $\mathcal{A}$ the linear order $<_{i_{0}}$. Recall $\mathcal{A}_{i_{0}}^{\prime}, \mathcal{A}_{i_{0}}^{\prime \prime}, I_{i_{0}}^{\prime}$, and $I_{i_{0}}^{\prime \prime}$ from 2.4. To each $i \in I_{i_{0}}^{\prime}$ (or $i \in I_{i_{0}}^{\prime \prime}$ ), we associate the weights $\alpha_{i}^{\prime}:=\alpha_{i}$ (resp. $\alpha_{i}^{\prime \prime}:=\sum \alpha_{k}$ where the sum runs over

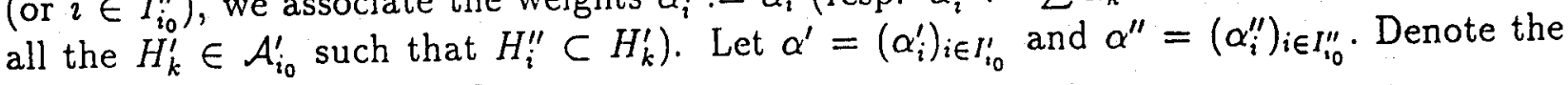
restriction of $f_{j}$ to $H_{i_{0}}$ by $\bar{f}_{j}$. Define

$$
U_{\alpha}^{\prime}=\prod_{j \in I_{10}^{\prime}} f_{j}^{\alpha^{\prime}}, U_{\alpha}^{\prime \prime}=\prod_{j \in I_{10}^{\prime \prime}} \bar{f}_{j}^{\alpha_{j}^{\prime \prime}} .
$$


Recall $I_{i_{0}}^{*}=\left\{i \in I_{i_{0}}^{\prime} \mid H_{i} \cap H_{i_{0}}=\emptyset\right\}$. Fix a branch of $\prod_{j \in I_{i 0}}\left(f_{j}^{\alpha}\right)_{\mid H_{i_{0}}}$ and call it $c_{i_{0}}$. Then $c_{i_{0}}$ is a constant number. Choose a branch of $U_{\alpha}^{\prime}$ and a branch of $U_{\alpha}^{\prime \prime}$ on each bounded chamber of $\operatorname{Ch}\left(\mathcal{A}_{i_{0}}^{\prime}\right)$ and on each bounded chamber of $\operatorname{Ch}\left(\mathcal{A}_{i_{0}}^{\prime \prime}\right)$ respectively. Also choose a branch of $f_{i_{0}}^{\alpha_{10}}$ on each bounded chamber $\Delta \in \operatorname{Ch}(\mathcal{A})$. Define a branch $U_{\Delta}$ of $U_{\alpha}$ on $\Delta \in \mathrm{Ch}(\mathcal{A})$ as follows (we use the terminology from the proof of Proposition 3.1.2):

(i) if $\Delta$ is undivided, then $\Delta \in \operatorname{Ch}\left(\mathcal{A}_{i_{0}}^{\prime}\right)$. Define $U_{\Delta}=\left(f_{i_{0}}^{\alpha_{i_{0}}}\right.$ on $\left.\Delta\right)\left(U_{\alpha}^{\prime}\right.$ on $\left.\Delta\right)$.

(ii) if $\Delta$ is the heir of $\Delta^{\prime} \in \operatorname{Ch}\left(\mathcal{A}_{i_{0}}^{\prime}\right)$, then define $U_{\Delta}=\left(f_{i_{0}}^{a_{i_{0}}}\right.$ on $\left.\Delta\right)\left(U_{\alpha}^{\prime} \text { on } \Delta^{\prime}\right)_{\mid \Delta}$.

(iii) if $\Delta$ is either a cutoff or newborn, then let $\Delta^{\prime \prime} \in \operatorname{Ch}\left(\mathcal{A}_{i_{0}}^{\prime \prime}\right)$ be the wall of $\Delta$. Choose a unique branch $U_{\Delta}^{\prime}$ of $U_{\alpha}^{\prime}$ on $\Delta$ such that $U_{\Delta \mid \Delta^{\prime \prime}}^{\prime}=c_{i_{0}}\left(U_{\alpha}^{\prime \prime}\right.$ on $\left.\Delta^{\prime \prime}\right)$. Let $U_{\Delta}=\left(f_{i_{0}}^{\alpha_{0}}\right.$ on $\left.\Delta\right) U_{\Delta}^{\prime}$.

Recall that we are using the $\beta$ nbc-orientation for every chamber of $\operatorname{Ch}\left(\mathcal{A}_{i_{0}}^{\prime}\right), \operatorname{Ch}\left(\mathcal{A}_{i_{0}}^{\prime \prime}\right)$ and $\operatorname{Ch}(\mathcal{A})$. Then

(i) if $\Delta \in \operatorname{Ch}(\mathcal{A})$ is the heir of $\Delta^{\prime} \in \operatorname{Ch}\left(\mathcal{A}_{i_{0}}^{\prime}\right)$, then the corresponding $\beta$ nbc-flags are equal. So the orientation of $\Delta$ is the induced one from the orientation of $\Delta^{\prime}$, and

(iii) if $\Delta \in \mathrm{Ch}(\mathcal{A})$ is either a cutoff or newborn, then the orthonormal frame for $\Delta$ is given by the orthonormal frame for $\Delta^{\prime \prime}:=\bar{\Delta} \cap H_{i_{0}} \in \operatorname{Ch}\left(\mathcal{A}_{i_{0}}^{\prime \prime}\right)$ together with the unit vector in the direction of $\Delta$ as the last vector of the frame.

Define $\mathrm{PM}_{i_{0}}(\mathcal{A}, \alpha), \operatorname{PM}\left(\mathcal{A}_{i_{0}}^{\prime}, \alpha^{\prime}\right)$ and $\operatorname{PM}\left(\mathcal{A}_{i_{0}}^{\prime \prime}, \alpha^{\prime \prime}\right)$ using these branches and orientations. We analytically continue the determinant $\operatorname{det} \mathrm{PM}_{i_{0}}(\mathcal{A}, \alpha)$ onto the hyperplane $\alpha_{i_{0}}=0$.

Proposition 5.3.1 Suppose that the real part of $\alpha_{i}$ is positive for all $i=1, \ldots, p, i \neq i_{0}$. Then

$$
\operatorname{det} \mathrm{PM}_{i_{0}}(\mathcal{A}, \alpha)_{\mid \alpha_{i_{0}}=0}=\operatorname{det} \mathrm{PM}\left(\mathcal{A}_{i_{0}}^{\prime}, \alpha^{\prime}\right) \operatorname{det} \mathrm{PM}\left(\mathcal{A}_{i_{0}}^{\prime \prime}, \alpha^{\prime \prime}\right) c_{i_{0}}^{\beta\left(\mathcal{A}_{i_{0}}^{\prime \prime}\right)} .
$$

Proof. Let $\Delta^{\prime} \in \operatorname{Ch}\left(\mathcal{A}_{i_{0}}^{\prime}\right)$ be a divided chamber. Let $\Delta^{+}$and $\Delta^{-}$be its heir and cutoff respectively. Let $U^{+}$and $U^{-}$be the branches of $U_{\alpha}$ on $\Delta^{+}$and $\Delta^{-}$. Choose a constant number $c_{\Delta^{\prime}}$ such that $U^{+}=c_{\Delta^{\prime}} U^{-}$on $\Delta^{\prime} \cap H_{i_{0}}$. Let $M$ be the matrix obtained from $\operatorname{PM}_{i_{0}}(\mathcal{A}, \alpha)$ by adding for each divided chamber $\Delta^{\prime} \in \operatorname{Ch}\left(\mathcal{A}_{i_{0}}^{\prime}\right)$ the column corresponding to the cutoff $\Delta^{-}$of $\Delta^{\prime}$ multiplied by $c_{\Delta^{\prime}}$ to the column corresponding to the heir $\Delta^{+}$of $\Delta^{\prime}$ and setting $\alpha_{i_{0}}=0$. Then $\operatorname{det} M=\operatorname{det} \mathrm{PM}_{i_{0}}(\mathcal{A}, \alpha)_{\mid \alpha_{i_{0}}=0}$. Note that the column of $M$ corresponding to $\Delta^{+}$is

$$
\left[\int_{\Delta^{\prime}} U_{\alpha}^{\prime} f_{i_{0}}^{\alpha_{i_{0}}} \phi_{1}, \ldots, \int_{\Delta^{\prime}} U_{\alpha}^{\prime} f_{i_{0}}^{\alpha_{i_{0}}} \phi_{\beta}\right]_{\left.\right|_{\alpha_{i_{0}}=0} ^{t}}^{t} .
$$

Write

$$
\mathrm{M}=\left[\begin{array}{ll}
P & Q \\
R & S
\end{array}\right]
$$

where $P$ is a square matrix of size $\beta\left(\mathcal{A}_{i_{0}}^{\prime}\right)$ and $S$ is a square matrix of size $\beta\left(\mathcal{A}_{i_{0}}^{\prime \prime}\right)$. Since the first $\beta\left(\mathcal{A}_{i_{0}}^{\prime}\right)$ columns of $M$ are labelled by $\operatorname{Ch}\left(\mathcal{A}_{i_{0}}^{\prime}\right)$, it follows from Lemma 2.4.10 that $P=\operatorname{PM}\left(\mathcal{A}_{i_{0}}^{\prime}, \alpha^{\prime}\right)$.

When computing $R$ and $S$ we may take $\phi=\Xi(B)$ where $B=\left(\nu B^{\prime \prime}, H_{i_{0}}\right) \in \overline{\beta \mathbf{n b c}}\left(\mathcal{A}_{i_{0}}^{\prime \prime}\right)$ with $B^{\prime \prime} \in \beta \operatorname{nbc}\left(\mathcal{A}_{i_{0}}^{\prime \prime}\right)$. Write $\phi=\psi\left(\alpha_{i_{0}} \omega_{i_{0}}\right)$. Let $\Delta^{\prime} \in \operatorname{Ch}\left(\mathcal{A}_{i_{0}}^{\prime}\right)$. Set $\Delta_{t}^{\prime}=\Delta^{\prime} \cap\left\{f_{i_{0}}=t\right\}$ 
and $F(t)=\int_{\Delta_{t}^{\prime}} U_{a}^{\prime} \psi$. Define real numbers $a<b$ such that $\Delta_{t}^{\prime} \neq \emptyset$ if and only if $a \leq t \leq b$. Using the variable $t=f_{i_{0}}$, Fubini's theorem and integration by parts give

$$
\pm \int_{\Delta^{\prime}} U_{\alpha}^{\prime} f_{i_{0}}^{\alpha_{i_{0}}} \phi=\int_{a}^{b} \alpha_{i_{0}} t^{\alpha_{i_{0}}-1} F(t) d t=\left[t^{\alpha_{i_{0}}} F(t)\right]_{a}^{b}-\int_{a}^{b} t^{\alpha_{i_{0}}} F^{\prime}(t) d t .
$$

Taking the limit as $\alpha_{i_{0}} \rightarrow 0, \Re a_{i_{0}}>0$, we get

$$
\lim \left[\left[t^{\alpha_{i_{0}}} F(t)\right]_{a}^{b}-\int_{a}^{b} t^{\alpha_{i_{0}}} F^{\prime}(t) d t\right]=\left\{\begin{array}{cc}
0 & 0 \notin\{a, b\} \\
F(0) & 0=a<b \\
-F(0) & a<b=0
\end{array}\right.
$$

If $\Delta^{\prime}$ is divided, then we apply the first part to get zero. If $H_{i_{0}}$ intersects $\bar{\Delta}^{\prime}$ in a face of codimension $>1$, then $F(0)=0$. If $H_{i_{0}}$ does not intersect $\bar{\Delta}^{\prime}$, then the integral is again zero. Thus $M\left(\Delta^{\prime}, \phi\right)=0$. This shows that $R=0$.

It remains to compute the entries of $S$. Let $\Delta \in \mathrm{Ch}(\mathcal{A})$ be either a cutoff or newborn. In this case $H_{i_{0}}$ is a wall of $\Delta$ so $\Delta_{0}=C^{\prime \prime}\left(B^{\prime \prime}\right)$. Let $\Delta^{\prime \prime}=C^{\prime \prime}\left(B^{\prime \prime}\right)$. It follows from Lemma 2.4 .11 that $\phi^{\prime \prime}:=\psi_{\mid \Delta^{\prime \prime}}=\Xi\left(B^{\prime \prime}, \mathcal{A}_{i_{0}}^{\prime \prime}\right)$. Set $\Delta_{t}=\Delta \cap\left\{f_{i_{0}}=t\right\}$ and $G(t)=\int_{\Delta_{t}} U_{\Delta}^{\prime} \psi$, where $U_{\Delta}^{\prime}$ is a unique branch of $U_{\alpha}^{\prime}$ on $\Delta$ such that $U_{\Delta \mid \Delta^{\prime \prime}}^{\prime \prime}=c_{i_{0}}\left(U_{\alpha}^{\prime \prime}\right.$ on $\left.\Delta^{\prime \prime}\right)$. Define real numbers $a<b$ such that $\Delta_{t} \neq \emptyset$ if and only if $a \leq t \leq b$. Then $0 \in\{a, b\}$. Recall the choice of branch of $U_{\alpha}$ on $\Delta$ and orientation of $\Delta$. By the same calculation as above, using the variable $t=f_{i_{0}}$, Fubini's theorem and integration by parts, we get

$$
\mathrm{M}(\Delta, \phi)=\lim \int_{\Delta} U_{\alpha} \phi=G(0)=c_{i_{0}} \int_{\Delta^{\prime \prime}} U_{\alpha}^{\prime \prime} \phi^{\prime \prime}=c_{i_{0}} M\left(\Delta^{\prime \prime}, \phi^{\prime \prime}\right)
$$

as $\alpha_{i_{0}} \rightarrow 0, \Re \alpha_{i_{0}}>0$. So $S=c_{i_{0}} \operatorname{PM}\left(\mathcal{A}_{i_{0}}^{\prime \prime}, \alpha^{\prime \prime}\right)$. Thus we have

$$
\operatorname{det} \mathrm{PM}_{i_{0}}(\mathcal{A}, \alpha)_{\mid \alpha_{i_{0}}=0}=\operatorname{det} \mathrm{M}=(\operatorname{det} P)(\operatorname{det} S)=\operatorname{det} \mathrm{PM}\left(\mathcal{A}_{i_{0}}^{\prime}, \alpha^{\prime}\right) \operatorname{det} \mathrm{PM}\left(\mathcal{A}_{i_{0}}^{\prime \prime}, \alpha^{\prime \prime}\right) c_{i_{0}}^{\beta\left(\mathcal{A}_{i_{0}}^{\prime \prime}\right)} \text {. }
$$

Corollary 5.3.2

$$
\operatorname{det} \mathrm{PM}_{i_{0}}^{*}(\mathcal{A}, \alpha)_{\mid \alpha_{i_{0}}=0}=\operatorname{det} \mathrm{PM}^{*}\left(\mathcal{A}_{i_{0}}^{\prime}, \alpha^{\prime}\right) \operatorname{det} \mathrm{PM}^{*}\left(\mathcal{A}_{i_{0}}^{\prime \prime}, \alpha^{\prime \prime}\right) c_{i_{0}}^{\beta\left(\mathcal{A}_{i_{0}}^{\prime \prime}\right)}
$$

Proof. When the real part of $\alpha_{i}$ is positive for all $i=1, \ldots, p, i \neq i_{0}$, this functional equality has been proved in Proposition 5.3.1. Therefore this equality holds true everywhere.

Remark 5.3.3 The recursion formula 5.3.1, in the case of arrangements in general position, is found in [V1, p.546]. 


\subsection{Proof of the main theorem}

We prove the theorem by induction on $(n, p)$ (equipped with lexicographical order). If $n=1$ the theorem is well-known. If $\beta(\mathcal{A})=0$, then the theorem asserts $1=1$. Note that $\beta(\mathcal{A})=0$ whenever $p \leq n$. Let $i_{0} \in I$. From the induction hypothesis we have

$$
\operatorname{det} \mathrm{PM}\left(\mathcal{A}_{i_{0}}^{\prime}, \alpha^{\prime}\right)=R\left(\mathcal{A}_{i_{0}}^{\prime}\right)^{\alpha^{\prime}} B\left(\mathcal{A}_{i_{0}}^{\prime}, \alpha^{\prime}\right)
$$

and

$$
\operatorname{det} \mathrm{PM}^{*}\left(\mathcal{A}_{i_{0}}^{\prime \prime}, \alpha^{\prime \prime}\right)=R\left(\mathcal{A}_{i_{0}}^{\prime \prime}\right)^{\prime \prime} B\left(\mathcal{A}_{i_{0}}^{\prime \prime}, \alpha^{\prime \prime}\right)
$$

First step : we determine the product of critical values.

The induction hypothesis gives, together with Corollary 5.3.2 and Corollary 4.2.3,

$$
\left.c_{1}^{\alpha_{1}} \ldots c_{p}^{\alpha_{p}}\right|_{\alpha_{i_{0}}=0}=\left.R(\mathcal{A})^{\alpha}\right|_{\alpha_{i_{0}}=0} .
$$

Thus we have $c_{k}^{\alpha_{k}}=\prod_{j=1}^{\beta} R\left(f_{k}, \Delta_{j}\right)^{\alpha_{k}}$ for $k \neq i_{0}$. By considering another linear order $<_{k}$ $\left(k \neq i_{0}\right)$, we have

$$
\left.c_{1}^{\alpha_{1}} \ldots c_{p}^{\alpha_{p}}\right|_{\alpha_{k}=0}=\left.R(\mathcal{A})^{\alpha}\right|_{\alpha_{k}=0}
$$

so $c_{i_{0}}^{\alpha_{i_{0}}}=\prod_{j=1}^{\beta} R\left(f_{i_{0}}, \Delta_{j}\right)^{\alpha_{i_{0}}}$.

Second step : we determine the rational function.

We have

$$
\operatorname{det} \mathrm{PM}_{i_{0}}^{*}(\mathcal{A}, \alpha)=R(\mathcal{A})^{\alpha} B(\mathcal{A}, \alpha) h_{i_{0}}(\alpha)
$$

First summarize what we know about the rational function $h_{i_{0}}$. Let

$$
\mathcal{L}=\left\{\alpha(F)+m \mid F \text { is a dense edge in } L\left(\mathcal{A}_{\infty}\right), m \in \mathbb{Z}\right\} .
$$

Lemma 5.4.1 (i) $h_{i_{0}}$ is independant (up to sign) of $i_{0}$.

(ii) The numerator and the denominator of $h$ are (up to sign) products of linear forms belonging to $\mathcal{L}$.

(iii) For all $i_{0} \in I,\left.h\left(\alpha_{1}, \cdots, \alpha_{p}\right)\right|_{\alpha_{i_{0}}=0}$ is equal to either 1 or -1 .

Proof. (i) follows from Proposition 3.3.5 and the fact that both $B(\mathcal{A}, \alpha)$ and $R(\mathcal{A})^{\alpha}$ are independent of $i_{0}$.

As for (ii), recall that the determinant of $\operatorname{PM}_{i_{0}}(\mathcal{A}, \alpha)$ takes a finite nonzero value at each $\alpha \notin \operatorname{Rsn}(\mathcal{A})$ by Remark 3.3.3. Neither of $B(\mathcal{A}, \alpha)$ or $R(\mathcal{A})^{\alpha}$ has a zero or pole at $\alpha \notin \operatorname{Rsn}(\mathcal{A})$ (cf. Remark 4.1.4). Therefore $h$ is a rational function which takes a finite nonzero value at every $\alpha \notin \operatorname{Rsn}$. Since $\operatorname{Rsn}(\mathcal{A})$ is the union of a locally finite infinite family of hyperplanes, we have (ii).

Lastly, (iii) is a consequence of the induction assumption, Corollary 5.3.2 and Corollary 4.2.3. 
Lemma $5.4 .2 h$ is equal to a constant function which is either 1 or -1 .

Proof. Suppose that $h$ is not constant. By Lemma 5.4.1(ii), we may write $h$ as a fraction whose denominator and numerator are both products of finitely many elements of

$$
\mathcal{L}=\left\{\alpha(F)+m \mid F \text { is a dense edge in } L\left(\mathcal{A}_{\infty}\right), m \in \mathbb{Z}\right\}
$$

Suppose $\alpha(F)+m$ appears in the expression. By Lemma 5.4.1 (iii), $\alpha(F)-\alpha_{j}+m$ also appears in the expression for each $j$ such that $\alpha_{j}$ appears in $\alpha(F)$. Also, $\alpha(F)+\alpha_{j}+m$ also appears in the expression for each $j$ such that $\alpha_{j}$ does not appear in $\alpha(F)$. Therefore, by repeately using these observations, we finally can conclude that $\sum_{i \in J} \alpha_{i}+m$ appears for every subset $J$ of $I$. In particular, $\alpha_{1}+\cdots+\alpha_{p-1}+m$ appears in the expression. This implies either (i) $F:=H_{1} \cap \cdots \cap H_{p-1}$ is dense and $I(F)=\{1,2, \ldots, p-1\}$, or (ii) $F_{\infty}:=\bar{H}_{p} \cap \bar{H}_{\infty}$ is dense and $\bar{I}\left(F_{\infty}\right)=\{p, \infty\}$. Since (ii) is a contradiction, (i) always occurs. In particular, $H_{1}, \ldots, H_{p-1}$ are dependent and there exists $j_{0} \in\{1, \ldots, p-1\}$ such that $F=H_{1} \cap \cdots \cap H_{j_{0}-1} \cap H_{j_{0}+1} \cap \cdots \cap H_{p-1}$. If $\mathcal{A}$ is central, there is nothing to prove. So we may assume $\emptyset=H_{1} \cap \cdots \cap H_{p}$. Thus

$$
\emptyset=H_{1} \cap \cdots \cap H_{p}=F \cap H_{p}=H_{1} \cap \cdots \cap H_{j_{0}-1} \cap H_{j 0+1} \cap \cdots \cap H_{p} .
$$

This implies that $\alpha_{1}+\cdots+\alpha_{j_{0}-1}+\alpha_{j_{0}+1}+\cdots+\alpha_{p}+m$ does not appear in the expression of $h$, which is a contradiction. This shows that $h$ is a constant. By Lemma 5.4.1 (iii), the constant is equal to either 1 or -1 .

It follows from Lemma 5.4.2 that

$$
\operatorname{det} \mathrm{PM}_{i_{0}}^{*}(\mathcal{A}, \alpha)= \pm R(\mathcal{A})^{\alpha} B(\mathcal{A}, \alpha)
$$

Let us determine the sign. It is known that the $\operatorname{sign}$ is plus when $n=1$ or $\beta(\mathcal{A})=0$. By Corollaries 5.3.2 and 4.2.3, we can inductively show that the sign is always plus:

$$
\operatorname{det} \mathrm{PM}_{i_{0}}^{*}(\mathcal{A}, \alpha)=R(\mathcal{A})^{\alpha} B(\mathcal{A}, \alpha) .
$$

This, together with Remark 3.3.3, proves the main theorem.

Remark 5.4.3 We could show in the same way that the periodic function $\varphi_{i_{0}}$ appearing in the proof of theorem 5.2 .1 is constant.

Remark 5.4.4 It should be interesting to study the connection between the roots of a Bernstein polynomial of $f=\left(f_{1}, \cdots, f_{p}\right)$ ([S]) and the poles of $\operatorname{det} \mathrm{PM}_{i_{0}}^{*}(\mathcal{A}, \alpha)$.

Acknowledgements. The first author thanks F. Loeser, C. Sabbah and F. Maaref for fruitful discussions. The second author also thanks K. Aomoto, P. Orlik, R. Silvotti and A. Varchenko for useful and stimulating discussions. 


\section{References}

[A] Aomoto, K.: Les équations aux différences finies et les intǵrales de fonctions multiformes, J. Fac. Sci. Tokyo 22 (1975), 271-297 et 26, 519-523 (1979)

[AK] Aomoto, K., Kita, M.: Hypergeometric functions (in Japanese). Tokyo: Springer 1994

[BV] Brylawski, T., Varchenko, A.: The determinant formula for a matroid bilinear form (preprint)

[FT] Falk, M. J., Terao, H.: $\beta$ nbc-bases for cohomology of local systems on hyperplanes complements, Trans. Amer. Math. Soc. (to appear)

[Ki] Kita, M.: On hypergeometric functions in several variables II. The wronskian of the hypergeometric functions of type $(n+1, m+1)$, J. Math. Soc. Japan 45, 645-689 (1993)

[Ko] Kohno, T.: Homology of a local system on the complement of hyperplanes, Proc. Japan Acad. 62, Ser. A, 144-147 (1986)

[L] Loeser, F.: Arrangements d'hyperplans et somme de Gauss, Ann. Sci. École Norm. Sup. 24, 379-400 (1991)

[LS] Loeser, F. and Sabbah, C.: Equations aux différences finies et déterminants d'intégrales de fonctions multiformes, Comment. Math. Helv. 66, 458-503 (1991)

[OT] Orlik, P., Terao, H.: Arrangements of hyperplanes. Grundlehren der Math. Wiss. 300, Berlin Heildelberg New York: Springer, 1992

[S] Sabbah, C.: Proximité évanescente I, Compositio Math. 62, 283-328 (1987)

[SV] Schechtman, V., Varchenko, A.: Arrangements of hyperplanes and Lie algebra homology, Invent. math. 106, 139-194 (1991)

[STV] Schechtman, V., Terao, H., Varchenko, A.: Local systems over complements of hyperplanes and the Kac-Kazhdan conditions for singular vectors, J. Pure Appl. Algebra 100, 93-102 (1995)

[V1,V2] Varchenko, A.: The Euler Beta-function, the Vandermonde determinant, Legendre's equation, and critical values of linear functions on a configuration of hyperplanes, Math. USSR Izvestija 35 (1990), 543-572 and 36, 155-168 (1991)

[V3] Varchenko, A.: Multidimensional hypergeometric functions and representation theory of Lie algebras and quantum groups, Advanced Series in Mathematical Physics - 21, World Scientific Publishers, 1995

[Z] Ziegler, G.: Matroid shellability, $\beta$-systems, and affine arrangements, J. Alg. Combinatorics, 1, 283-300 (1992) 